Ann. Biol. anim., Bioch. Biophys., I967, 7 (I), 33-38.

\title{
LA SIGNIFICATION DU pH DANS LE TUBE DIGESTIF DE GALLUS DOMESTICUS
}

\author{
Claire HERPOL et G. VAN GREMBERGEN \\ Laboratoire de Zoophysiologie, Université de Gand (Belgique) \\ H. 1. K. W., 35, Ledeganckstraat, Gand (Belgique)
}

\section{SOMMAIRE}

Le $\mathrm{pH}$ des différentes régions du système digestif de poulets mâles du type White Leghorn a été déterminé in vivo et post mortem sur 450 sujets d'âges différents examinés à jeun.

L'ensemble des résultats a été soumis à l'analyse statistique.

L'efficacité du processus de digestion étant entre autre déterminée par l'influence importante que le $\mathrm{pH}$ exerce sur l'action enzymatique, la fonction de chaque organe du tractus digestif est examinée dans cette optique.

L'évolution du $\mathrm{pH}$ dans les organes successifs du système digestif, un point qui donne lieu à de nombreuses controverses dans la littérature, a été établie d’après nos données.

\section{INTRODUCTION}

L'importance de la connaissance du degré d'acidité ou d'alcalinité dans les divers organes constituant l'appareil digestif des oiseaux n'échappe certes à personne. De nombreux chercheurs s'y sont intéressés et nous avons nous-mêmes publié dans cette revue un article à ce sujet (HERPol et VAN GREMBERGEN, I96I).

Depuis lors, nous avons accumulé un nombre considérable de résultats expérimentaux qui, soumis à une analyse statistique, nous permet de tirer des conclusions plus générales.

\section{MATÉRIEL E'T MÉTHODES}

Ayant établi (HERPoL, I966) que l'âge des sujets n'a pas une influence significative sur le pH dans le tube digestif, nous avons été amenés à grouper les chiffres obtenus, sur des sujets d'âge différent dans des conditions expérimentales par ailleurs identiques. Il s'agit de $45^{\circ}$ poulets White 
Leghorn (W.L.) de sexe mâle, examinés à jeun. Nous avons écarté les sujets de moins d'un jour d'âge, parce que les mesures post mortem dans l'estomac de tels individus sont imprécises pour des raisons opératoires (HER POL, 1966).

La méthode de mesure a été décrite antérieurement (Herpol et VAN Grembergen, I96I ; HerPOL, I966).

\section{RÉSULTATS}

Le résultat global de l'analyse statistique de ces données est reproduit dans le tableau I. L'ensemble des déterminations y est présenté à l'aide des grandeurs suivantes :

$n=$ nombre de mesures ;

$\bar{x}=$ moyenne arithmétique $\mathrm{du} \mathrm{pH} ;$

$\sigma=$ écart-type de la distribution ;

$\sigma_{\bar{x}}=$ erreur standard de la moyenne;

extrêmes $=$ étendue réelle de la distribution ;

$\bar{x}_{\text {н-ion }}=$ moyenne du $\mathrm{pH}$ calculée par l'intermédiaire de la concentration en ions d'hydrogène (pour permettre la comparaison avec certaines données de la littérature).

\section{TABLEAU I}

Le pH dans le systeme digestif de Gallus domesticus (W. L. ; $\delta$; à jeun)

\begin{tabular}{|c|c|c|c|c|c|c|c|}
\hline \multirow{2}{*}{ Organe } & \multirow{2}{*}{$n$} & \multirow{2}{*}{$\bar{x}$} & \multicolumn{2}{|c|}{$\begin{array}{l}\text { Paramètres de } \\
\text { la distribution }\end{array}$} & \multicolumn{2}{|c|}{ Extrêmes } & \multirow{2}{*}{$\bar{x}_{\text {H-ion }}$} \\
\hline & & & $\sigma$ & $\sigma_{\bar{x}}$ & $\min$. & $\max$. & \\
\hline \multicolumn{8}{|l|}{ In vivo } \\
\hline Jabot..,$\ldots \ldots \ldots$ & 450 & 6,4 & 0,56 & 0,03 & 4,2 & 7,8 & 5,9 \\
\hline Estomac $\ldots . . \ldots$ & 450 & 1,4 & 0,49 & 0,02 & 0,2 & 3,1 & 1,2 \\
\hline \multicolumn{8}{|l|}{ Post mortem } \\
\hline Jabot. & 450 & 6,3 & 0,77 & 0,04 & 4,0 & 7,8 & 5,5 \\
\hline Estomac glandulaire & 450 & 1,8 & 0,57 & 0,03 & 0,3 & 4,1 & 1,5 \\
\hline Gésier $\ldots \ldots \ldots \ldots \ldots$ & 450 & 2,5 & 0,71 & 0,03 & 0,4 & 5,4 & 2,0 \\
\hline Duodénum. & 450 & 6,4 & 0,31 & 0,01 & 5,2 & 7,6 & 6,3 \\
\hline Iléum I,. & 450 & 6,6 & 0,41 & 0,02 & 5,5 & 7,7 & 6,4 \\
\hline Iléum II..... & 450 & 7,2 & 0,39 & 0,02 & 5,7 & 8,2 & 7,0 \\
\hline Cæcum.... & 450 & 6,9 & 0,36 & 0,02 & 5,7 & 8,1 & 6,8 \\
\hline Rectum........... & 450 & 7,0 & 0,48 & 0,02 & 5,4 & 8,4 & 6,7 \\
\hline Bile prélevée dans & & & & & & & \\
\hline la vésicule $\ldots \ldots \ldots$ & 327 & 6,6 & 0,45 & 0,02 & 5,3 & 7,8 & 6,4 \\
\hline
\end{tabular}

\section{DISCUSSION}

Il est sans doute inutile de souligner l'importance de l'écart-type de la distribution pour l'interprétation des résultats. Ces données confirment en grandes ligne $e_{S}$ 
nos premiers résultats de I96I (HERPOL et VAN GREMBERGEN), basés sur une centaine de mesures diverses. Quelle est leur signification dans le cadre du processus de digestion?

Le rôle essentiel du jabot est de constituer un réservoir pour la nourriture. Il est peu probable que cet organe joue un rôle important dans la digestion proprement dite. Quoique certains auteurs (TEICHMANN, I889; SchwarZ et TELIER, I923) 1ui attribuent une telle fonction, cette thèse nous semble difficile à soutenir.

Il n'est évidemment pas impossible que le jabot puisse dans certains cas contenir de la pepsine. Elle peut très certainement être régurgitée jusque dans le jabot, même en quantité appréciable, mais elle y trouve des conditions de $\mathrm{pH}(\bar{x}=6,4$ in vivo; $\bar{x}=6,3$ post mortem) extrêmement défavorables à son action. Par contre, ces conditions peuvent être considérées comme favorables à l'action des carbohydrases dont le $\mathrm{pH}$ optimal d'activité se situe aux environs de la neutralité. Il est d'ailleurs possible de mettre en évidence, dans des conditions expérimentales, une certaine dégradation par ces enzymes (qui proviennent fort probablement de micro-organismes ou de l'extérieur) dans le jabot (MANGolD, I929, p. 24 ; BolTON, I962 ; IvORECSzyLIT et al., I965). Nous doutons cependant qu'une telle action puisse prendre d'importantes proportions dans des conditions de nutrition normales, puisque les graines ne subissent aucune destruction mécanique avant leur passage par le gésier et puisque leur enveloppe leur assure une protection considérable.

Le gésier semble être de toute évidence le siège du début de la dégradation enzymatique des constituants de la nourriture, puisque celle-ci ne fait que passer par l'estomac glandulaire dont la fonction paraît être uniquement sécrétoire.

Le rôle du gésier est donc double : d'une part son action mécanique, d'autre part sa participation à la digestion comme site d'actions enzymatiques. Parmi les divers ferments présents dans le contenu du gésier, la pepsine semble être seule à $\mathrm{y}$ trouver des conditions de $\mathrm{pH}$ vraiment favorables $(\bar{x}=2,5$ post mortem). La protéinase active en milieu acide, contenue dans les extraits d'estomacs glandulaires de quelques espèces d'oiseaux, présente en effet un maximum d'activité aux pH les plus acides : $\mathrm{pH}$ I,o et $\mathrm{pH}$ 2,0 (HERPOL, I964). L'activité optimale de la dipeptidase, qui est la principale exopeptidase formée par l'estomac glandulaire, se situe, par contre, aux environs du pH 8,5 (DE Rycke, r960) et la lipase, présente aussidans le gésier, n'est plus active aux valeurs de $\mathrm{pH}$ inférieures à 4,9 (HEwITT et SchELKopF, I955). Ces derniers auteurs ont démontré que l'amylase, également présente dans le contenu du gésier, commence a être active à partir et au-dessus du $\mathrm{pH} 3,6$. Une très légère activité dans ce domaine nous semble donc acceptable. GroEBBELS (I930) a effectivement démontré à l'aide de fistules, une digestion de carbohydrates au niveau du gésier, malheureusement sur une autre espèce d'oiseau, notammentl'oie.

L'intestin des oiseaux est 1'organe où se situe la majeure partie de la digestion enzymatique. Tel est l'avis de divers auteurs (ex. : MANGoL, I929, p. 67 ; HEWITT et SCHELKOPF, I955). Le $\mathrm{pH}$ dans les diverses régions intestinales est donc particulièrement important pour le conditionnement de l'activité des sucs digestifs qui doivent y exercer leur action spécifique.

La plupart des enzymes présents dans l'intestin agissent de façon optimale en milieu légèrement alcalin. Nitzan et Alumor (I960) déterminent l'activité de la trypsine pancréatique aux environs du $\mathrm{pH} 7,6$, tandis que l'activité optimale de la 
dipeptidase se situe aux alentours du $\mathrm{pH}$ 8,5 (DE RyckE, I96I), de la maltase autour du $\mathrm{pH}$ 6,9 (LAws et MOORE, rg63) et de la lipase au $\mathrm{pH}$ 7,0 (HEwitT et SCHELKOPF, 1955). Ces derniers enregistrent également une activité de diverses carbohydrases dans 1'intervalle de $\mathrm{pH}$ compris entre 4,9 et 8,5 . Ces divers enzymes trouvent donc dans l'intestin un milieu favorable à leur action respective (duodénum $\bar{x}=6,4$; iléum I $\bar{x}=6,6$; iléum II $\bar{x}=7,2$ ).

Le tôle des cæcums des oiseaux reste l'objet de diverses prises de position que nous ne pouvons pas approfondir ici. Nous nous bornerons à constater qu'il y règne un $\mathrm{pH}(\bar{x}=6,9)$ qui permettrait une certaine activité des divers enzymes qui passent dans ces organes avec le contenu de l'intestin grêle et à remarquer que les phases majeures de la digestion sont de toute façon terminées à cet endroit.

La réaction de la bile du poulet est légèrement acide, ceci à l'encontre de celle des mammifères phytophages, qui présente une réaction alcaline. Nous notons cependant une valeur plus élevée $\left(\bar{x}=6,6 ; \bar{x}_{\mathrm{H}-\mathrm{ion}}=6,4\right)$ que celle de FARNER (I942) 5,88

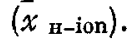

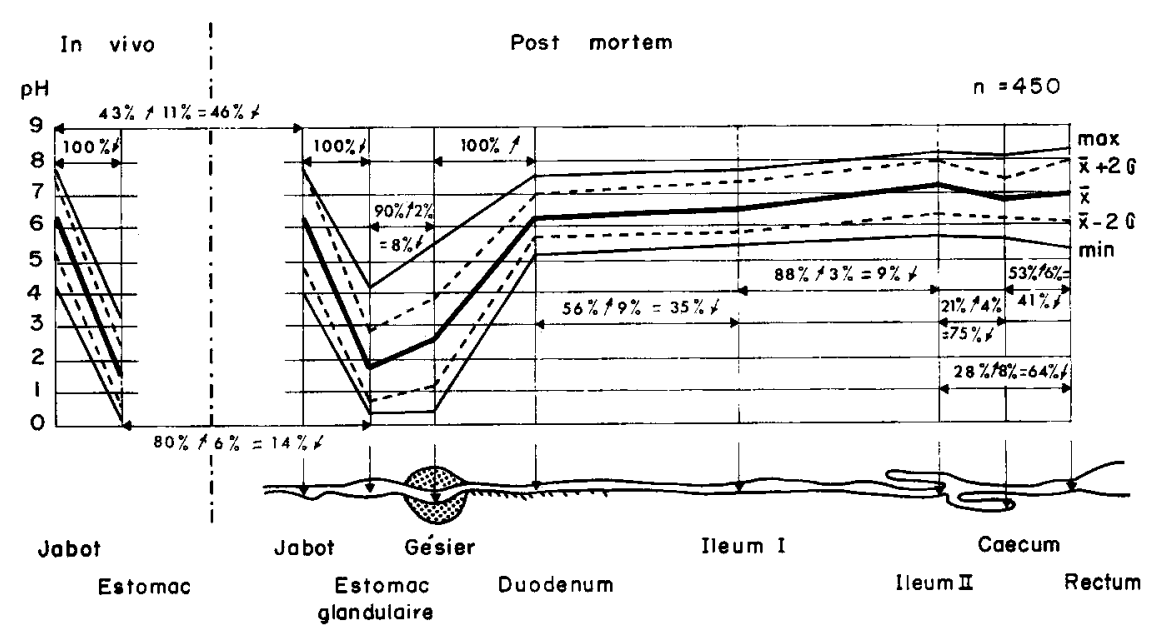

Fig. 1. - Évolution $d u$ pH dans le tube digestif de Gallus domesticus.

Un autre point qui donne lieu à de nombreuses controverses est l'évolution du $\mathrm{pH}$ dans les organes successifs du système digestif du poulet. Selon FarNER (I942) la valeur $\mathrm{du} \mathrm{pH}$ augmente à partir du duodénum pour diminuer dans les régions terminales (iléum II et rectum) de l'intestin. HEWITT et SchELKOPF (I955) sont, par contre, d'avis que le $\mathrm{pH}$ continue son augmentation à partir du duodénum, pour atteindre sa valeur la plus élevée dans le rectum. WINGET et al. (I962) partagent cette opinion. Orson et MANN (I935) et HEWITT et SCHELIKOPF (I955) déterminent dans les cæcums une acidité supérieure à celle des parties environnantes (iléum et rectum) de l'intestin.

L'évolution du $\mathrm{pH}$ dans les organes successifs du système digestif d'après nos données est reproduite sur la figure $I$ et les tendances en ressortent nettement. Il est entendu que les droites qui relient les points successifs sur la figure ne signifient nullement une évolution linéaire du $\mathrm{pH}$ dans l'intervalle entre les points de mesure, 
le tracé ayant seulement pour but d'illustrer clairement l'évolution générale. De plus; nous avons cru devoir contrôler si ces tendances évolutives correspondent à la réalité en pointant pour chaque individu l'augmentation ou la diminution du $\mathrm{pH}$ entre les mesures successives. La concordance entre la direction du tracé et la variation du $\mathrm{pH}$ chez nos $45^{\circ}$ individus est dès lors clairement exprimée en pourcentages d'augmentation, de diminution ou d'égalité des valeurs du $\mathrm{pH}$, tels qu'ils sont indiqués sur la figure $I$.

La détermination de tels pourcentages nous a également permis de constater que le $\mathrm{pH}$ de la bile est plus élevé que celui du duodénum en $60 \mathrm{p}$. Ioo des cas (3I p. Ioo de diminution) et que celui de la première partie de l'iléum en $54 \mathrm{p}$. Ioo des cas (39 p. Ioo de diminution). Nous ne pouvons donc pas nous ranger du côté de FARNER (I942) qui constate, se basant sur une vingtaine de mesures, que le pH de l'intestin est toujours plus élevé que le $\mathrm{pH}$ de la bile, prélevée dans la vésicule.

Notons en guise de conclusion que la figure I peut valoir comme le résumé des résultats de nos recherches, puisque nous y avons indiqué, en dehors de la moyenne arithmétique du $\mathrm{pH}(\bar{x})$, les limites de l'intervalle de sécurité, correspondant à une probabilité de $95 \mathrm{p}$. Ioo $(\mathrm{P}=0,05)$, soit $\bar{x} \pm 2 \sigma$.

Reçu pour publication en octobre 1966.

\section{SUMMARY}

\section{AN INTERPRETATION OF $\mathrm{PH}$ VALUES IN THE DIGESTIVE TKACT OF THE DOMESTIC HEN}

$\mathrm{pH}$ values were determined according to the method previously described, on 450 digestive tracts of White Leghorn male chickens of various ages having been unfed for 24 hours (HERPOL and VAN GREMBERGEN, 196r ; HERPOL, 1966). The results were statistically analyzed ; they are summarized in table $\mathrm{I}$.

The prime role of the crop is to heap up food. Although digestive enzymes, such as pepsin or carbohydrates, may be present in crop contents, they cannot have any considerable activity : the $\mathrm{pH}$ is not favourable to pepsin activity $(\bar{x}=6.4$ in vivo $; 6.3$ post mortem $)$; and were it favourable to some carbohydrates, one must nor forget that the grains are not yet mechanically destroyed and therefore remain protected. functions.

Since food quickly passes through the proventriculus, it is clear that this organ has but secretory

The gizzard thus appears to be the first important site of enzyme activity-apart from its role in crushing the grain. The $\mathrm{pH}$ value $(\tilde{x}=2.5$ post mortem) is favourable to pepsin activity ( $\mathrm{pH}$ optimum r.o to 2.o) (HERPOL, 1964). but it is unfavourable to other enzymes recorded in gizzard contents : $\mathrm{pH}$ optimum of dipeptidase 8.5. (DE RrckE, I960); lipase and amylase activities at $\mathrm{pH}$ values superior to 4.9 and 3.6 respectively. (HEwITT and SCHELKOPF, I955). However, a low amylase activity is not unlikely.

The intestine is the most important organ for enzyme activity. $\mathrm{pH}$ conditions ( $\mathrm{pH}$ values in duodenum 6.4 ; in ileum I: 6.6 ; in ileum II : 7.2) are near the optima of a series of enzymes present in intestine contents : $\mathrm{pH}$ optimum of trypsine 7.6 (NITZAN and Alumot, 1960); of dipeptidase 8.5 (DE RYCKE, I960); of maltase 6.9 (LAWS and MOORE, 1963); of lipase 7.0; and of different carbohydrases whose $\mathrm{pH}$ optima range between 4.9 and 8.5 (HEWITT and SCHELKOPF, I955).

The function of the cæcum has been discussed for a long time, but is still not accurately defined. $\mathrm{pH}$ value in the cæcum (6.9) might allow the enzyme activity to go on, though the digestion is almost completed in the intestine.

The chicken's bile is slightly acid ( $\mathrm{pH} \vec{x}=6.6$ ). 
Another point under controversy is the altering of $\mathrm{pH}$ values in the different parts of digestive tract. Figure $\mathrm{I}$ shows this evolution according to our results. The percents were calculated individually; they tally with the pattern of the graph. However the linear variations do not represent the actual evolution of the $\mathrm{pH}$ values from one point to another. Figure $\mathbf{r}$ is in fact a diagrammatized summary of our results, including the means $(\bar{x})$ and the deviations $(\bar{x} \pm 2 \sigma)$.

\section{RÉFÉRENCES BIBLIOGRAPHIQUES}

Bolton W., I962. Digestion in the crop of the fowl. Proc. Nutr. Soc. 21, XXIV.

DE Rxcke P., 1960. Experimenteel onderzoek over exopeptidasen bij vogels. Thèse, Université de Gand (Belgique).

DE RyCKe P., 196r. Onderzoek over exopeptidasen bij het kuiken. Natuurwet. Tijdschr., 43, 82-86.

FarNer D. S., 1942. The hydrogen-ion concentration in avian digestive tracts. Poult Sci., 21, 445-450.

Grokbbets F., 1930. Die Verdauung bei der Hausgans, untersucht mit der Methode der Dauerkanule. Pfiuger's Arch. ges. Physiol., 224, 687-701.

Herpol C., Van Grembergen G., ig6r. Le $\mathrm{pH}$ dans le tube digestif des oiseaux. Ann. Biol,anim., Bioch., Biophys., 1, 31 7-321.

HERPOL C., I964. Activité protéolytique de l'appareil gastrique d'oiseaux granivores et carnivores. Ann. Biol. anim., Bioch., Biophys., 4, 239-244.

Herpol C., Ig66. Influence de l'âge sur le $\mathrm{pH}$ dans le tube digestif de Gallus domesticus. Ann Biol. anim., Bioch., Biophys., 6, 495-502.

HewitT E. H., ScheLKOPF L., 1955. pH-values and enzymatic activity of the digestive tract of the chicken. Ann. J. Vet. Res., 16, 576-579.

Ivorec-Szylit O., Mercier C., Raibaud P., Calet C., 1965. Contribution à l'étude de la dégradation des glucides dans le jabot du coq. Influence du taux de glucose du régime sur l'utilisation de l'amidon. $C$. R. Acad. Sci. Paris, 261, 3201-3203.

LAws B. M., Moore J. H., I963. Some observations on the pancreatic amylase and intestinal maltase of the chick. Canad. J. Bioch., 41, 2107-221 I.

MANGOLD E., 1929. Handbuch der Ernährung und des Sloffwechsels der Landwirlschaftlichen Nutztiere als Grundlagen der Fütterungslehre, vol. 2. Verlag von J. Springer, Berlin.

Nitzan Z., Alumot E., 1960. Proteolytic activity of chick's intestine. Bull. Res. Counc. Israel Ser. A, 9, 23-27.

Olson C. jr, Mann F. C., 1935. The physiology of the cæcum of the domestic fowl. J. Am. Vet. Med. Assoc., 8\%, $\mathrm{I}_{51-\mathrm{I} 59}$.

Schwarz C., Teller H., I923. Beiträge zur Physiologie der Verdauung. VIII. U̇ber die Kropfverdauung der Haushuhnes. Fermentforsch., 7, 254-269.

Teichmann M., 1889. Der Kropf der Taube. Arch. Mikr. Anat., 34, 235-247.

Winget C. M., Ashton G. C., Cawley A. J., I962. Changes in gastrointestinal pH associated with fasting in the laying hen. Poult. Sci., 41, III5-1120. 